Научная статья

УДК 616.458.72-008.61-07-089

DOI: https://doi.org/10.17816/brmma73193

\title{
ЭНДОГЕННЫЙ ГИПЕРКОРТИЗОЛИЗМ: ДОСТИЖЕНИЯ И ПЕРСПЕКТИВЫ В ДИАГНОСТИКЕ И ЛЕЧЕНИИ
}

\author{
Н.А Майстренко ${ }^{1}$, П.Н. Ромащенко ${ }^{1}$, В.Ю. Черебилло ${ }^{2}$, В.С. Довганюк' \\ ${ }^{1}$ Военно-медицинская академия имени С.М. Кирова, Санкт-Петербург, Россия \\ ${ }^{2}$ Первый Санкт-Петербургский государственный медицинский университет имени И.П. Павлова, Санкт-Петербург, Россия
}

Резюме. Изучены результаты обследования и лечения 647 больных эндогенным гиперкортизолизмом: кортикотропинома гипофиза выявлена у $494(76,4 \%)$ пациентов, кортикостерома и прекортикостерома надпочечника у $142(21,9 \%)$, двухсторонняя макроузелковая гиперплазия надпочечников первично надпочечникового генеза у 11 (1,7\%). Дифференциальная диагностика клинических форм эндогенного гиперкортизолизма основывалась на оценке уровня адренокортикотропного гормона и кортизола как в периферической крови, так и при селективном двустороннем заборе крови из надпочечниковых вен и нижних каменистых синусов, изучении характера проб с 8 мг дексаметазона. Топическая диагностика заключалась в оценке состояния надпочечников и гипофиза при компьютерной томографии и магнитно-резонансной томографии с применением контрастных препаратов, а использование специального программного обеспечения 3D-техники объемного рендеринга позволяло оптимизировать тактические и технические подходы к выполнению оперативных вмешательств. Тотального удаления кортикотропиномы гипофиза, по данным контрольной магнитно-резонансной томографии, удалось достичь в 92,3\% случаев, субтотального - в 7,7\%. Однако гормональной ремиссии удалось достичь только в 82,4\% наблюдений. Всем больным кортикостеромой и прекортикостеромой надпочечника выполнена адреналэктомия: у 6 открытым способом, у $136-$ эндовидеохирургическим (у 11 - лапароскопическим, у 124 - ретроперитонеоскопическим способом и у 1 больного выполнена торакоскопическая трансдиафрагмальная адреналэктомия. У всех больных операция привела к выздоровлению. Больные, страдающие доброкачественной макроузловой гиперплазией надпочечников, нуждались в консервативном лечении блокаторами стероидогенеза. Показания к хирургическому лечению в объеме односторонней адреналэктомии возникли у 9 больных.

Ключевые слова: эндогенный гиперкортизолизм; кортикотропинома; кортикостерома; дифференциальная диагностика; катетеризация каменистых синусов; транссфеноидальная резекция гипофиза; эндовидеохирургическая адреналэктомия.

Как цитировать:

Майстренко Н.А, Ромащенко П.Н., Черебилло В.Ю., Довганюк В.С. Эндогенный гиперкортизолизм: достижения и перспективы в диагностике и лечении // Вестник Российской военно-медицинской академии. 2021. Т. 23, № 3. С. 9-16. DOI: https://doi.org/10.17816/brmma73193 
Scientific article

DOI: https://doi.org/10.17816/brmma73193

\title{
ENDOGENOUS HYPERCORTICISM: ACHIEVEMENTS AND PROSPECTS IN DIAGNOSIS AND TREATMENT
}

\author{
N.A. Maistrenko', P.N. Romashchenko', V.Yu. Cherebillo², V.S. Dovganyuk' \\ ${ }^{1}$ Military Medical Academy named after S.M. Kirov, Saint Petersburg, Russia \\ ${ }^{2}$ Pavlov State Medical University of the Ministry of Health of the Russian Federation, Saint Petersburg, Russia
}

\begin{abstract}
The results of examination and treatment of 647 patients with endogenous hypercortisolism were studied: pituitary corticotropinoma was detected in 494 (76.4\%) patients, corticosteroma and pre - corticosteroma of the adrenal gland - in 142 (21.9\%), bilateral macro-nodular hyperplasia of the adrenal glands of primary adrenal origin-in 11 (1.7\%). Differential diagnosis of clinical forms of endogenous hypercortisolism was based on the assessment of the level of adrenocorticotropic hormone and cortisol, both in peripheral blood, and with selective bilateral blood sampling from the adrenal veins and lower stony sinuses, and the study of the nature of samples with $8 \mathrm{mg}$ of dexamethasone. Topical diagnostics consisted in assessing the state of the adrenal glands and pituitary gland during computed tomography and magnetic resonance imaging with the use of contrast agents, and the use of special software 3D-Volume Rendering Technique allowed optimizing tactical and technical approaches to performing surgical interventions. Of the operated patients with adrenocorticotropic hormone dependent endogenous hypercortisolism, total removal according to the control magnetic resonance imaging was achieved in $92.3 \%$ of cases, subtotal - in $7.7 \%$. However, hormonal remission was achieved only in $82.4 \%$ of cases. All patients with corticosteroma and pre-corticosteroma of the adrenal gland underwent adrenalectomy: in 6 patients by open method, in 136 patients by endovideosurgical method (in 11 patients by laparoscopic method, in 124 patients by retroperitoneoscopic method, and in 1 patient by thoracoscopic transdiaphragmatic adrenalectomy). In all patients, the operation led to recovery. Patients with benign macronodular hyperplasia of the adrenal glands needed conservative treatment with steroidogenesis blockers. Indications for surgical treatment in the volume of unilateral adrenalectomy occurred only in 2 patients.
\end{abstract}

Keywords: endogenous hypercortisolism; corticotropinoma; corticosteroma; differential diagnosis; stony sinus catheterization; transsphenoidal pituitary resection; endovideosurgical adrenalectomy.

To cite this article:

Maistrenko NA, Romashchenko PN, Cherebillo VYu, Dovganyuk VS. Endogenous hypercorticism: achievements and prospects in diagnosis and treatment. Bulletin of the Russian Military Medical Academy. 2021;23(3):9-16. D0l: https://doi.org/10.17816/brmma73193 


\section{ВВЕДЕНИЕ}

В этом году отмечается 180-летний юбилей одной из старейших хирургических клиник России - клиники факультетской хирургии им. С.П. Федорова Военномедицинской академии им. С.М. Кирова, основанной Н.И. Пироговым в 1841 г. С тех пор многие поколения ученых нашей кафедры, среди которых были выдающиеся хирурги с мировым именем, обогатили разные области хирургии. В том числе следует отметить их вклад в развитие эндокринной хирургии и, в частности, в изучение и лечение эндогенного гиперкортизолизма (ЭГ).

Родоначальником этого раздела эндокринной хирургии в России является великий хирург, ученый и педагог Сергей Петрович Федоров, имя которого носит наша клиника. Достоверным историческим фактом является то, что первую в России операцию на гипофизе, трансназальную гипофизэктомию, по поводу аденомы передней его доли с развитием акромегалии выполнил С.П. Федоров в 1914 г. в нашей клинике.

В период руководства С.П. Федоровым кафедральным коллективом с 1903 по 1936 г. до 20\% операций составляли вмешательства на эндокринных органах. Развитие эндокринной хирургии в ВМА было столь успешным, что в 1926 г. клинику тогда еще госпитальной хирургии посетил в знак уважения и с познавательной целью выдающийся американский хирург и ученый William Mayo. Сотрудники клиники активно интересовались достижениями ведущих ученых того времени, занимавшихся проблемами хирургической эндокринологии. В частности, В.Н. Шамов проходил стажировку в клинике известного американского нейрохирурга Х.У. Кушинга [1].

В.М. Ситенко, принявший кафедру от В.Н. Шамова, продолжил разработку научных материалов по хирургии надпочечников. В самом начале руководства кафедрой факультетской хирургии (в 1961 г.) В.М. Ситенко выполнил первую в России двустороннюю адреналэктомию. Следующий период в развитии эндокринной хирургии на кафедре, руководимой профессором А.И. Нечаем, характеризовался внедрением малоинвазивных технологий обследования больных, страдающих ЭГ (ультразвуковое исследование - УзИ, компьютерная томография - КТ, магнитно-резонансная томография - МРТ), многочисленными научными публикациями, в которых отражены результаты исследований, проводимых совместно с сотрудниками кафедр нейрохирургии, рентгенологии, патанатомии, анестезиологии и др.

Изучение результатов исследований, проведенных в клинике, дали основание сделать вывод о наличии общего патогенеза опухолевых и неопухолевых изменений в надпочечниках при ЭГ. Полученные данные подтвердили предположения академика В.Г. Баранова, сформулированное в 1955 г., о том, что гиперплазия, аденома и рак коркового слоя надпочечника могут быть проявлениями единого процесса. Показано, что с углублением болезни морфологические изменения очагового характера (опухоль), а возможно и вся ткань надпочечников, могут приобретать функциональную «самостоятельность», что функциональная значимость новообразований коркового вещества надпочечников неодинакова. Опухоль, обнаруживаемая в надпочечнике, не всегда обусловливает проявления ЭГ. При выборе метода лечения данного синдрома эти особенности необходимо учитывать особо с целью исключения «автономного» функционирования надпочечников, не пораженных опухолью [3]. Полученные данные нашли свое отражение в диссертационных исследованиях В.М. Трофимова (1986), А.Г. Вавилова (1992), Л.М. Краснова (1993), а монография «Синдром Иценко - Кушинга» (1991) под ред. В.Г. Баранова и А.И. Нечая стала настольной книгой для эндокринологов и хирургов, которая определила стратегию обследования и лечения больных ЭГ на многие годы [2-5].

Успехи, достигнутые за последние 25 лет под руководством академика Российской академии наук профессора Н.А. Майстренко совместно с сотрудниками кафедры нейрохирургии, в лечении ЭГ включают: современные иммунохимические исследования гормонов, лучевые методики, эндовидеохирургические вмешательства на гипофизе и надпочечниках, протонное облучение и др. [6-8]. При этом транссфеноидальная резекция гипофиза, лапароскопическая, ретроперитонеоскопическая и торакофреноскопическая адреналэктомия выполнены сотрудниками академии впервые в России [9].

Несмотря на совершенствование способов лечения и появление новых диагностических технологий, до настоящего времени вызывает трудности дифференциальная диагностика основных клинических форм ЭГ. Более чем у 50\% больных причина заболевания устанавливается со значительным опозданием. Нередко допускаются грубые тактические ошибки: длительное неэффективное консервативное лечение, выполнение патогенетически необоснованных адреналэктомий. По сообщениям многих авторов, число пациентов, которым выполняют нейрохирургические операции при «магнитно-резонансно-негативной» картине заболевания, не имеет тенденции к уменьшению, невзирая на достижения современной инструментальной диагностики. Отсутствие доказательной базы и обоснованного выбора методики лечения этих больных приводит к развитию рецидивов, а в ряде случаев, после двусторонней адреналэктомии, к хронической надпочечниковой недостаточности $[4,10,11]$.

Цель исследования - уточнить рациональные варианты лечения больных ЭГ на основании достоверной верификации его основных клинических форм с помощью современных диагностических технологий. 


\section{МАТЕРИАЛЫ И МЕТОДЫ}

Проанализированы результаты обследования и лечения 647 больных ЭГ со сроками наблюдения от 5 до 10 лет. Возраст больных на момент проведения операции колебался от 14 до 68 лет, составив в среднем $32,5 \pm 4,8$ лет. Значительную часть из них составили женщины. Большинство пациентов $(60,8 \%)$ находилось в активном (в трудовом отношении) возрасте от 21 до 50 лет. Клинические симптомы ЭГ были однотипными. Самыми частыми были: ожирение, дитрофические изменения кожного покрова, кардиомиопатия, артериальная гипертензия, остеопороз, диабет, вторичный иммунодефицит и др.

Для первичной диагностики ЭГ использовали стандартизованные методики: определение свободного кортизола в моче, определение кортизола в слюне в 23 ч, малую пробу с дексаметазоном (1 мг). После подтверждения ЭГ проводили дифференциальную диагностику между зависимой от адренокортикотропного гормона (АКТГ) и независимой от АКТГ формами заболевания, определяя уровень кортизола, кортизона, а также АКТГ и его ритм (в 8 и 23 ч) в плазме крови, изучали характер проб с 8 мг дексаметазона. Пациентам с высокой клинической предсказуемостью результата, чтобы ускорить диагностику, одновременно выполняли два биохимических теста [12, 13].

В нашей работе мы учитывали результаты исследований некоторых авторов о том, что вечерний тест подавления дексаметазоном недостаточно достоверен. Как известно, у некоторых больных АКТГ-зависимой формой ЭГ дексаметазон подавляет выработку кортизола, а у многих пациентов без заболевания - нет, особенно если они болеют другими острыми или хроническими заболеваниями, депрессией или являются алкоголиками. Все перечисленные заболевания активируют систему «гипоталамус - гипофиз - надпочечники» в результате стресса и вызывают резистентность к подавлению секреции кортизола дексаметазоном. Из-за таких сложностей при начальном скрининговом тесте на ЭГ больному может потребоваться повторное обследование через некоторое время [14, 15].

Для исключения субклинического гиперкортизолизма исследовали уровни предшественников глюкокортикоидов в периферической крови (кортикостерона, 11-дезоксикортикостерона, 11-дезоксикортизола). В моче определяли экскрецию свободного кортизола и свободного кортизона методом высокоэффективной жидкостной хроматографии с изучением хроматографических профилей кортикостероидов [16, 17].

Нейровизуализацию проводили с помощью МРТ от 1,5 до 3 Тл. Данный алгоритм был выполнен 493 пациентам, кроме одного, у которого были противопоказания к выполнению МРТ и диагноз подтверждался результатами КТ и забором крови из каменистых синусов [18].
При сомнениях в источнике гиперпродукции АКТГ выполняли селективную ангиографию с забором крови из кавернозных или нижних каменистых синусов. Оценивался градиент уровня АКТГ центр периферия и сторона поражения. Забор крови был выполнен 112 пациентам, диагноз АКТГ-зависимого ЭГ подтвержден [18]. У 32 пациентов при отсутствии нейровизуализации опухоли при МРТ либо сомнительных данных (при дифдиагностике рецидива или послеоперационных изменений) дополнительно выполнены позитронно-эмиссионная и компьютерная томографии. Очаг гиперпродукции был подтвержден. Пациенты впоследствии прооперированы.

Топическая диагностика новообразований надпочечников проводилась с использованием КТ и МРТ с введением контрастных препаратов, а применение специального программного обеспечения 3D-Volume Rendering Technique позволяло оптимизировать тактические и технические подходы к выполнению оперативных вмешательств [19]. Завершающим этапом диагностики являлось морфологическое и иммуногистохимическое исследование удаленных опухолей надпочечников и гипофиза [20, 21].

\section{РЕЗУЛЬТАТЫ И ИХ ОБСУЖДЕНИЕ}

После установления диагноза ЭГ проводили поиск причины заболевания. Изучение результатов клиникогормональных исследований и изменений в надпочечниках и гипофизе (КT, MPT) при первоначальном обследовании позволило установить следующие формы ЭГ у наблюдаемых больных: АКТГ-зависимая форма у 494 (76,4\%), АКТГ-независимая форма (макроузелковая форма заболевания надпочечников первично-надпочечникового генеза) - у 11 (1,7\%), кортикостерома надпочечника - у 142 (21,9\%) пациентов.

Диагноз АКТГ-зависимого гиперкортизолизма устанавливали согласно общепринятым рекомендациям [21]. Раннее проведенные нами $[5,16,20]$ исследования показали, что при определении уровней гормонов надпочечников ключевыми критериями для постановки диагноза являлись определение уровня АКТГ, кортизола в плазме крови и исследование их суточного ритма, а также проведение большой пробы (8 мг/сут) с дексаметазоном. Для больных АКТГзависимой формой ЭГ характерными были повышенные уровни АКТГ - 31,8 $\pm 3,6$ пмоль/л утром (норма 2,2-10 пмоль/л) и 28,4 \pm 4,2 пмоль/л вечером, кортизола $-668,4 \pm 36,7$ нмоль/л утром (норма 150-650 нмоль/л) и 616,9 $\pm 39,3$ нмоль/л вечером (норма 25-270 нмоль/л) соответственно. Установлено, что после проведения большой дексаметазоновой пробы на 3-и сутки уровень кортизола в плазме крови и суточная экскреция 17-оксикортикостероидов (ОКС) с мочой снижались на $50 \%$ и более. Характер гормональных нарушений и функциональной пробы свидетельствовали об АКТГ-зависимой 
форме ЭГ и необходимости выявления кортикотропиномы гипофиза.

Основными методами топической диагностики являлись КТ и МРТ гипофиза и надпочечников. При КТ в надпочечниках в большинстве случаев выявляли двустороннюю гиперплазию, а в гипофизе - аденому.

Однако, несмотря на применение в последние годы современных компьютерных томографов, сохраняются трудности диагностики микроаденом гипофиза (менее 1 см в диаметре). КТ не позволяет дифференцировать небольшие патологические новообразования, рентгенологическая плотность которых близка к плотности ликвора или нормальной мозговой ткани. Сложности трактовки получаемой информации обусловлены рядом объективных причин. Близость костных образований дает значительное количество наводок и артефактов. Повышенная васкуляризация гипофиза, наличие в непосредственной близи кавернозных синусов, внутренних сонных артерий и вилизиева круга не дают возможности рассчитывать на достоверную информацию даже при КТ с внутривенным введением контрастных препаратов. Следовательно, малые размеры аденом гипофиза и объективные недостатки получаемой информации не позволяют в настоящее время считать КТ оптимальным методом диагностики этой патологии (чувствительность не более 70\%) [21].

Из прооперированных пациентов тотальное удаление кортикотропиномы гипофиза, по данным контрольной МРТ, удалось достичь в 92,3\% случаев, субтотальное в 7,7\%. Однако гормональной ремиссии удалось достичь только в $82,4 \%$ наблюдений. Полученные результаты нейрохирургического лечения свидетельствуют о том, что не всегда тотальное удаление аденомы гипофиза, по данным МРТ, сопровождается полной гормональной ремиссией. Вероятно, при инвазивном характере роста остаются микрофрагменты образования в кавернозном синусе, которые не видны и находятся за пределами разрешающей способности МРТ. Нередко поражение носит мультифокальный характер, и удаление большего манифестного очага не всегда приводит к выздоровлению пациента.

Данные гормональных исследований у больных кортикостеромой надпочечника характеризовались нормальными уровнями АКТГ плазмы крови $-8,5 \pm 2,7$ пмоль/л утром и 5,2 $\pm 3,1$ пмоль/л вечером. В то же время уровень кортизола был повышен $-840,7 \pm 85,4$ нмоль/л утром и 819,6 $\pm 74,1$ нмоль/л вечером. Суточная экскреция с мочой 17-ОКС составила 58,6 \pm 9,5 мкмоль/л утром. Проведение большой дексаметазоновой пробы позволило установить, что на 3-и сутки уровень кортизола в плазме крови и суточная экскреция 17-ОКС с мочой снижались менее чем на 50\%. При этом патологических изменений в гипофизе при МРТ с внутривенным введением контрастных препаратов не выявлялось. Полученные лаборатороно-инструментальные данные доказывали наличие функциональной автономии на уровне надпочечников и опухоли. Выполнение КТ и МРТ позволяло выявить новообразование надпочечника с чувствительностью 92,5 и 100\% соответственно. Полученные данные совпадают с данными других исследователей [21].

Всем больным этой группы выполнена адреналэктомия: у 6 - открытым способом, у $136-$ эндовидеохирургическим (у 11 - лапароскопическим, у 124 - ретроперитонеоскопическим способом и у 1 выполнена торакоскопическая трансдиафрагмальная адреналэктомия). Анализ результатов эндовидеохирургических вмешательств показал очевидные преимущества ретроперитонеоскопической адреналэктомии, при которой не было осложнений и переходов на открытую операцию. При этом доступе нет необходимости мобилизации внутренних органов, а центральная вена надпочечника выделяется до его мобилизации, что особенно важно при удалении гормонально-активных опухолей.

Лапароскопическая адреналэктомия представляется менее эффективной, особенно слева. При данной локализации опухоли надпочечника интра- и послеоперационные осложнения развились у каждого 2-го больного, а в двух случаях потребовалась конверсия [11, 20].

Экспериментальные данные и накопленный практический опыт свидетельствуют о необходимости более широкого использования эндовидеохирургических вмешательств на надпочечниках, которые оказались эффективными в 87,3\% случаев. Исключение составляют больные с опухолями размером больше 8 см и признаками злокачественного роста.

В 3 наблюдениях адреналэктомия оказалась неэффективной (гистологически - светлоклеточная аденома и внеопухолевая гиперплазия коры надпочечника). У этих пациентов в отдаленные сроки после операции (5 лет и более) наблюдалось сохранение клинической картины ЭГ с гиперпродукцией кортизола $-845 \pm 28,7$ нмоль/л утром и $664 \pm 26,5$ нмоль/л вечером. При проведении пробы с 8 мг дексаметазона у них наблюдалось подавление продукции кортизола крови более чем на $50 \%$. Наряду с этим обращено внимание на повышение уровня АКТГ плазмы крови до 16,8 $\pm 3,4$ пмоль/л утром и 12,3 $\pm 3,6$ пмоль/л вечером. Повторные МРТ гипофиза с внутривенным введением контрастного препарата позволили выявить микроаденому. Следовательно, первоначальный диагноз кортикостеромы надпочечника у этих больных изменен на АКТГ-зависимый ЭГ. После проведения протонотерапии на область выявленной микроаденомы гипофиза у всех пациентов наступило выздоровление.

Особую группу составили больные, у которых отмечался нормальный уровень АКТГ плазмы крови 9,1 $\pm 2,1$ пмоль/л утром и 4,6 $\pm 2,8$ пмоль/л вечером, повышенный уровень кортизола - 712,1 $\pm 27,8$ нмоль/л утром и 684,6 $\pm 31,3$ нмоль/л вечером. Суточная экскреция с мочой 17-ОКС составила 46,4 $\pm 6,1$ мкмоль/л утром [22]. Большая дексаметазоновая проба была 
отрицательной, суточная экскреция 17-ОКС с мочой снизились не менее чем на $50 \%$ от фонового уровня. При выполнении КТ у этих больных в надпочечниках опухоль не выявлялась, а отмечалась их макроузелковая двусторонняя гиперплазия. При выполнении МРТ с внутривенным введением контрастного препарата аденом в гипофизе не выявлено. Полученные данные свидетельствовали об АКТГ-независимой форме ЭГ. С целью уменьшения проявлений ЭГ у этих пациентов выполнена адреналэктомия: односторонняя - у 9 больных со стороны наиболее измененного надпочечника, двусторонняя у 2 больных. После односторонней адреналэктомии у всех больных отмечена непродолжительная ремиссия заболевания с последующим его прогрессированием, а после двусторонней адреналэктомии развился синдром Нельсона. При морфологическом исследовании удаленных надпочечников выявлялись признаки макроузелковой гиперплазии.

Наряду с этим отмечено повышение уровня АКТГ крови до 18,2 \pm 4,8 пмоль/л утром (норма 2,2-10 пмоль/л) и 15,3 \pm 4,1 пмоль/л вечером у больных после как односторонней, так и двусторонней адреналэктомии. При повторных МРТ гипофиза у этих пациентов выявлена микроаденома гипофиза (кортикотропинома). После транссфеноидального удаления микроаденомы гипофиза признаки ЭГ регрессировали. Анализ полученных результатов обследования и лечения больных позволил пересмотреть первоначальный диагноз макроузелковой гиперплазии надпочечников первично-надпочечникового генеза и констатировать у этих пациентов кортикотропиному гипофиза.

Таким образом, представленный анализ результатов обследования и хирургического лечения 647 пациентов
ЭГ позволил установить окончательный диагноз АКТГзависимой формы заболевания у 505 (78,1\%) больных, кортикостеромы - у 142 (19,9\%).

\section{ЗАКЛЮЧЕНИЕ}

Представленные диагностические трудности выявления основных клинических форм ЭГ очевидны несмотря на совершенствование лабораторных и инструментальных методов исследования. Подавление секреции кортизола крови после пробы с 8 мг дексаметазона более чем на $50 \%$ и нормальный уровень АКТГ плазмы, отсутствие опухоли в надпочечниках или их двусторонняя макроузелковая гиперплазия диктуют целенаправленный поиск микроаденомы гипофиза с помощью высокопольной прицельной МРТ с использованием контрастных парамагнитных средств.

Основной целью лечения больных ЭГ является нормализация уровней гормонов коры надпочечников, и как следствие, уменьшение клинических проявлений гиперкортизолизма. При этом, важное значение имеет раннее выполнение хирургического вмешательства до развития тяжелых осложнений, которые определяют неблагоприятный прогноз. Соблюдение диагностического алгоритма позволит при ЭГ своевременно выявлять кортикотропиному и выполнять эффективную транссфеноидальную аденомэктомию, а при кортикостероме адреналэктомию. Такой подход, который может быть реализован только в условиях специализированного стационара, обеспечивает рациональную лечебную тактику, хорошие результаты лечения и высокое качество жизни пациентов.

\section{СПИСОК ЛИТЕРАТУРЫ}

1. Майстренко Н.А. Владимир Николаевич Шамов (1882-1962) // Вестник хирургии им. И.И. Грекова. 2012. Т. 171, № 6. С. 9-12.

2. Майстренко Н.А., Ромащенко П.Н., Алиев А.К. Научная школа профессора С.П. Федорова. СПб.: Гуманистика, 2020. 263 с.

3. Калинин А.П., Майстренко Н.А. Хирургия надпочечников. М.: Медицина, 2000. 214 с.

4. Калинин А.П., Майстренко Н.А., Ветшев П.С. Хирургическая эндокринология: руководство. СПб.: Питер, 2004. 941 с.

5. Майстренко Н.А., Вавилов А.Г., Довганюк В.С. Современные аспекты хирургии надпочечников // Хирургия. 2000. № 5. С. 21-26. 6. Steffensen C., Bak A.M., Rubeck K.Z. Epidemiology of Cushing's syndrome // Neuroendocrinology. 2010. Vol. 92, No. 1. P. 1-5. DOI: 10.1159/000314297

7. Hirsch D., Tsvetov G., Manisterski Y., et al. Incidence of Cushing's syndrome in patients with significant hypercortisoluria // Eur J Endocrinol. 2017. Vol. 176, No. 1. P. 41-48. DOI: 10.1530/EJE-16-0631
8. Valassi E., Franz H., Brue T., et al. ERCUSYN Study Group. Diagnostic tests for Cushing's syndrome differ from published guidelines: data from ERCUSYN // Eur J Endocrinol. 2017. Vol. 176, No. 5. P. 613-624. DOI: 10.1530/EJE-16-0967

9. Ромащенко П.Н. Обоснование доступов при эндовидеохирургических вмешательствах на надпочечниках. (клинико-анатомическое исследование): дис. ... канд. мед. наук. СПб., 2000. Режим доступа: https://search.rsl.ru/ru/record/01000264722 Дата обращения: 09.07.21.

10. Новообразования надпочечников / под ред. В.Д. Федорова М.: Медпрактика, 2002.

11. Эндовидеохирургия надпочечников / под ред. Н.А. Майстренко СПб. ЭЛБИ, 2003.

12. Lacroix A., Feelders R.A., Stratakis C.A., et al. Cushing's syndrome // Lancet. 2015. Vol. 386, No. 9996. P. 913-927. DOI: 10.1016/S0140-6736(14)61375-1 
13. Pivonello R., Leo M.De., Cozzolino A., et al. The treatment of Cushing's disease // Endocr Rev. 2015. Vol. 36, No. 4. P. 385-486. DOl: 10.1210/er.2013-1048

14. Chan K.C., Lit L.C., Law E.L., et al. Diminished urinary free cortisol excretion in patients with moderate and severe renal impairment // Clin Chem. 2004. Vol. 50, No. 4. P. 757-769. DOI: 10.1373/clinchem.2003.029934

15. Alwani R.A., Schmit Jongbloed L.W., de Jong F.H., et al. Differentiating between Cushing's disease and pseudo-Cushing's syndrome: comparison of four tests // Eur J Endocrinol. 2014. Vol. 170, No. 4. P. 477-486. DOI: 10.1530/EJE-13-0702

16. Майстренко Н.А., Довганюк В.С., Фомин Н.Ф. «Гормональнонеактивные» опухоли надпочечников. СПб.: ЭЛБИ-СПб, 2001.

17. Fassnacht M., Arlt W., Bancos I., et al. Management of adrenal incidentalomas: European Society of Endocrinology Clinical Practice Guideline in collaboration with the European Network for the Study of Adrenal Tumors // Eur J Endocrinol. 2016. Vol. 175, No. 2. P. 1-34. DOI: 10.1530/EJE-16-0467

18. Гуссаова Н.В., Савелло А.В., Цой У.А. и др. Опыт применения катетеризации пещеристых и нижних каменистых си- нусов в дифференциальной диагностике АКТГ-зависимого гиперкортицизма // Трансляционная медицина. 2015. № 2-3. С. 31-32.

19. Ромащенко П.Н., Железняк И.С., Блюмина С.Г. и др. КТ-планирование доступа для адреналэктомии // Вестник Российской военно-медицинской академии. 2019. № 1. C. 105-109.

20. Довганюк В.С. Современные технологии в диагностике и хирургическом лечении новообразований надпочечников: дис. ... док. мед. наук. СПб., 2005. Режим доступа: https://www. dissercat.com/content/sovremennye-tekhnologii-v-diagnostike-ikhirurgicheskom-lechenii-novoobrazovanii-nadpochechn Дата обращения: 09.07.21.

21. Болезнь Иценко-Кушинга: клиника, диагностика, лечение: практическое руководство для врачей / под ред. И.И. Дедова, Г.А. Мельниченко. М.: УП Принт, 2012.

22. Майстренко Н.А. Проблемные аспекты диагностики и хирургического лечения синдрома Иценко-Кушинга // Вестник хирургии им. И.И. Грекова. 2010. Т. 154, № 3. С. 49-56.

\section{REFERENCES}

1. Maystrenko NA. Vladimir Nikolayevich Shamov. (1882-1962). Grekov's Bulletin OF Surgery. 2012;171(6):9-12. (In Russ.).

2. Maystrenko NA, Romashchenko PN, Aliyev AK. Nauchnaya shkola professora S.P. Fedorova. Saint Petersburg: Gumanistika; 2020. 263 p. (In Russ.).

3. Kalinin AP, Maystrenko NA, Khirurgiya nadpochechnikov. Moscow: Meditsina; 2000. 214 p. (In Russ.).

4. Kalinin AP, Maystrenko NA, Vetshev PS. Khirurgicheskaya endokrinologiya: rukovodstvo. Saint Petersburg: Piter; 2004. 941 p. (In Russ.).

5. Maystrenko NA, Vavilov AG, Dovganyuk VS. Sovremennyye aspekty khirurgii nadpochechnikov. Khirurgiya. 2000;5:21-26. (In Russ.).

6. Steffensen C, Bak AM, Rubeck KZ. Epidemiology of Cushing's syndrome. Neuroendocrinology. 2010;92(1):1-5. DOI: 10.1159/000314297

7. Hirsch D, Tsvetov G, Manisterski Y, et al. Incidence of Cushing's syndrome in patients with significant hypercortisoluria. Eur J Endocrinol. 2017;176(1):41-48. D0I: 10.1530/EJE-16-0631

8. Valassi E, Franz H, Brue $T$, et al. ERCUSYN Study Group. Diagnostic tests for Cushing's syndrome differ from published guidelines: data from ERCUSYN. Eur J Endocrinol. 2017;176(5): 613-624. DOI: 10.1530/EJE-16-0967

9. Romashchenko PN. Obosnovaniye dostupov pri endovideokhirurgicheskikh vmeshatelstvakh na nadpochechnikakh. (kliniko-anatomicheskoye issledovaniye): [dissertation] Saint Peterburg; 2000. 24 s. (In Russ.). Available from: https://search.rsl. $\mathrm{ru} / \mathrm{ru} / \mathrm{record} / 01000264722$

10. Novoobrazovaniya nadpochechnikov. VD. Fedorova editor. Moscow: Medpraktika; 2002. (In Russ.).

11. Endovideokhirurgiya nadpochechnikov. N.A. Maystrenko editor. SPb.: ELBI; 2003. (In Russ.).
12. Lacroix A, Feelders RA, Stratakis CA, et al. Cushing's syndrome. Lancet. 2015;386(9996):913-27. DOI: 10.1016/S0140-6736(14)61375-1

13. Pivonello R, Leo MDe, Cozzolino A, et al. The treatment of Cushing's disease. Endocr Rev. 2015;36(4):385-486. DOI: 10.1210/er.2013-1048

14. Chan KC, Lit LC, Law EL, et al. Diminished urinary free cortisol excretion in patients with moderate and severe renal impairment. Clin Chem. 2004;50(4):757-769. DOI: 10.1373/clinchem.2003.029934 15. Alwani RA, Schmit Jongbloed LW, de Jong FH, et al. Differentiating between Cushing's disease and pseudo-Cushing's syndrome: comparison of four tests. Eur J Endocrinol. 2014;170(4):477-86. DOI: 10.1530/EJE-13-0702

16. Maystrenko NA, Dovganyuk VS, Fomin NF. Gormonalnoneaktivnyye opukholi nadpochechnikov. Saint Petersburg: ELBI-SPb; 2001. (In Russ.).

17. Fassnacht M, Arlt W, Bancos I, et al. Management of adrenal incidentalomas: European Society of Endocrinology Clinical Practice Guideline in collaboration with the European Network for the Study of Adrenal Tumors. Eur J Endocrinol. 2016;175(2):1-34. DOI: 10.1530/EJE-16-0467

18. Gusaaova NV, Savello AV, Tsoy UA, et al. Experience in the use of combined cavernous and inferior petrosal sinus sampling in the differential diagnostic of acth-dependent cushing's syndrome. Translational Medicine. 2015;(2-3):31-32). (In Russ.).

19. Romashchenko PN, Zheleznyak IS, Blyumina SG, et al. Ct-planning access for adrenalectomy. Bulletin of the Russian Military Medical Academy. 2019;1:105-109. (In Russ.).

20. Dovganyuk VS. Sovremennyye tekhnologii v diagnostike i khirurgicheskom lechenii novoobrazovaniy nadpochechnikov. [dissertation] Saint Petersburg; 2005. (In Russ.) Available from: https://www. 
dissercat.com/content/sovremennye-tekhnologii-v-diagnostike-ikhirurgicheskom-lechenii-novoobrazovanii-nadpochechn

21. Bolezn Itsenko-Kushinga: klinika, diagnostika, lecheniye: prakticheskoye rukovodstvo dlya vrachey. Dedova II, Melnichenko GA, editors. Moscow: UP Print; 2012. (In Russ.).

\section{ОБ ABTOPAX}

*Виталий Сафронович Довганюк, доктор медицинских наук, доцент; e-mail: vit.dov65@rambler.ru; ORCID: 0000-0002-0038-795X; SPIN-код: 2713-3684

Павел Николаевич Ромащенко, доктор медицинских наук, професcop; e-mail: romashchenko@rambler.ru;

ORCID: 0000-0001-8918-1730; SPIN-код: 3850-1792

Николай Анатольевич Майстренко, доктор медицинских наук, профессор; e-mail: nik.m.47@mail.ru; ORCID: 0000-0002-1405-7660; SPIN-код: 2571-9603

Всеволод Юрьевич Черебилло, доктор медицинских наук, профессор; e-mail: cherebillo@mail.ru;

ORCID: 0000-0001-6803-9954; SPIN-код: 2887-6943
22. Maystrenko NA. Problem aspects of diagnostics and surgical treatment of itsenko-cushing syndrome. Grekov's Bulletin of Surgery. 2010;154(3):49-56. (In Russ.).

\section{AUTHORS INFO}

*Vitaly S. Dovganyuk, doctor of medical sciences, associate professor; e-mail: vit.dov65@rambler.ru;

ORCID: 0000-0002-0038-795X; SPIN code: 2713-3684

Pavel N. Romashchenko, doctor of medical sciences, professor; e-mail: romashchenko@rambler.ru; ORCID: 0000-0001-8918-1730; SPIN code: 3850-1792

Nicolay A. Maistrenko, doctor of medical sciences, professor; e-mail: nik.m.47@mail.ru; ORCID: 0000-0002-1405-7660; SPIN code: 2571-9603

Vsevolod Yu. Cherebillo, doctor of medical sciences, professor; e-mail: cherebillo@mail.ru; ORCID: 0000-0001-6803-9954; SPIN code: 2887-6943

* Автор, ответственный за переписку / Corresponding author 